\title{
Initial experience with a novel EUS-guided core biopsy needle (SharkCore): results of a large North American multicenter study
}

Authors

Institutions
Christopher J. DiMaio ${ }^{1}$, Jennifer M. Kolb ${ }^{1}$, Petros C. Benias ${ }^{2}$, Hiral Shah ${ }^{3}$, Shashin Shah ${ }^{3}$, Oleh Haluszka ${ }^{4}$, Jennifer Maranki $^{4}$, Kaveh Sharzehi ${ }^{4}$, Eric Lam ${ }^{5}$, Stuart R. Gordon ${ }^{6}$, Sarah M. Hyder ${ }^{6}$, Pavlos Z. Kaimakliotis ${ }^{7}$, Satya B. Allaparthi ${ }^{7}$, Frank G. Gress ${ }^{8}$, Amrita Sethi ${ }^{8}$, Ashish R. Shah ${ }^{8}$, Jose Nieto ${ }^{9}$, Vivek Kaul ${ }^{10}$, Shivangi Kothari ${ }^{10}$, Truptesh H. Kothari ${ }^{10}$, Sammy Ho ${ }^{11}$, Manhal J. Izzy ${ }^{11}$, Neil R. Sharma ${ }^{12}$, Rabindra R. Watson ${ }^{13}$, V. Raman Muthusamy ${ }^{13}$, Douglas K. Pleskow ${ }^{14}$, Tyler M. Berzin ${ }^{14}$, Mandeep Sawhney ${ }^{14}$, Emad Aljahdi ${ }^{14}$, Marvin Ryou ${ }^{15}$, Clarence K. Wong ${ }^{16}$, Parantap Gupta ${ }^{17}$, Dennis Yang', Susana Gonzalez ${ }^{1}$, Douglas G. Adler ${ }^{18}$

Institutions are listed at the end of article. submitted

14. December 2015

accepted after revision

5. July 2016

\section{Bibliography}

DOI http://dx.doi.org/

10.1055/s-0042-112581

Published online: 30.8.2016

Endoscopy International Open

2016; 04: E974-E979

(c) Georg Thieme Verlag KG

Stuttgart · New York

E-ISSN 2196-9736

\section{Corresponding author}

Christopher J. DiMaio, MD,

FASGE

Dr. Henry D. Janowitz Division of

Gastroenterology

Icahn School of Medicine at

Mount Sinai

One Gustave L. Levy Place, Box 1069

New York, NY 10029

Fax: +1-212-241-2276

Christopher.

DiMaio@mountsinai.org
Background and aims: The ability to safely and effectively obtain sufficient tissue for pathologic evaluation by using endoscopic ultrasound (EUS) guidance remains a challenge. Novel designs in EUS needles may provide for improved ability to obtain such core biopsies. The aim of this study was to evaluate the diagnostic yield of core biopsy specimens obtained using a novel EUS needle specifically designed to obtain core biopsies.

Patients and methods: Multicenter retrospective review of all EUS-guided fine-needle biopsies obtained using a novel biopsy needle (SharkCore FNB needle, Medtronic, Dublin, Ireland). Data regarding patient demographics, lesion type/location, technical parameters, and diagnostic yield was obtained.

Results: A total of 250 lesions were biopsied in 226 patients (Median age 66 years; 113 (50\%) male). Median size of all lesions (mm): 26 (2-

\section{Introduction}

$\nabla$

EUS-guided tissue acquisition (EUS-TA) can be performed by way of fine-needle aspiration (EUS-FNA) or fine-needle biopsy (EUS-FNB), otherwise known as core biopsy. The most critical issue in performing EUS-FNA is that of the non-diagnostic procedure. This not only results in higher costs related to repeat procedures and/or attempts at alternative biopsy methods, but also patient angst related to delayed diagnosis of a potentially malignant lesion. EUS-FNB is commonly utilized as a salvage technique in cases where EUS-FNA fails to procure adequate tissue. In addition, these core biopsy specimens are critical in making an accurate diagnosis in certain disease states, such as lymphoma and autoimmune pancreatitis.

Interest in EUS-FNB is growing. From a clinical practice standpoint, the ability to reliably obtain adequate diagnostic tissue with minimal effort and without the need for rapid on-site evaluation
150). Overall, a cytologic diagnosis was rendered in $81 \%$ specimens with a median number of 3 passes. When rapid onsite cytologic evaluation (ROSE) was used, cytologic diagnostic yield was $126 / 149$ (85\%) with a median number of 3 passes; without ROSE, cytologic diagnostic yield was 31/ $45(69 \%, P=0.03)$ with a median number of 3 passes. Overall, a pathologic diagnosis was rendered in 130/147 (88\%) specimens with a median number of 2 passes. Pathologic diagnostic yield for specific lesion types: pancreas 70/81 (86\%), subepithelial lesion $13 / 15$ (87\%), lymph node $26 / 28$ (93\%). Ten patients (10/226, $4 \%$ ) experienced adverse events: 4 acute pancreatitis, 5 pain, 1 fever/ cholangitis.

Conclusions: Initial experience with a novel EUS core biopsy needle demonstrates excellent pathologic diagnostic yield with a minimum number of passes.

of the specimen would allow for more efficient and less costly procedures. In addition, there is a movement toward use of EUS-guided core biopsy specimens to perform molecular and genetic studies to aid in both diagnosis and guide therapy of pancreatic neoplasms [1,2].

Currently, there is no standard or preferred needle type, size, or FNB technique that can serve this function. Novel designs in needles may provide an advantage in allowing for the acquisition of diagnostic tissue with minimal needle passes, thus making on-site cytologic analysis obsolete. In this study, we report the initial North American experience of using a novel core biopsy needle in EUS-TA.

\section{Patients and methods}

$\nabla$

This study is a multicenter retrospective review of all patients who underwent EUS-TA performed by using a novel core biopsy needle (SharkCore FNB 


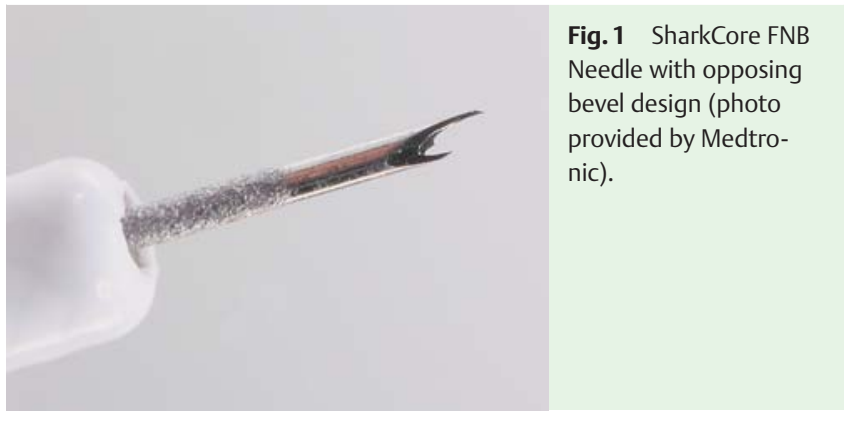

needle, Medtronic, Dublin, Ireland). The needle tip is designed with 6 cutting-edge surfaces and an opposing bevel designed to catch tissue as it is sheared off ( $\bullet$ Fig. 1). A total of 18 centers participated, consisting of academic tertiary referral centers and high-volume community-based medical centers. Institutional Review Board approval was obtained from all participating centers prior to data collection and analysis.

The study time frame was between October 2014 and March 2015. The study involved patients referred for EUS-TA of a lymph node, solid mass lesion involving the gastrointestinal lumen, pancreas, liver, or biliary system, or any other extraluminal masses suitable for EUS-TA. Use of FNA, FNB, or both for any particular lesion was left to the discretion of each individual endoscopist at each center.

Data regarding patient demographics, target lesion type and location, technical parameters, diagnostic yield, and adverse events were obtained.

\section{Equipment and technique}

The SharkCore FNB needle was utilized for all tissue acquisition in this study. In this study, EUS-guided biopsy was performed using a 25-gauge needle and/or a 22-gauge needle. The needle size used for each patient was left to the discretion of the endoscopist.

All procedures were performed with the stylet in place within the needle. Upon advancing the needle out of the scope channel and sheath, the stylet was retracted a few millimeter's prior to puncturing the target lesion. Care was taken to avoid retracting the stylet prior to needle advancement out of the sheath so as to avoid inadvertent "catching" and puncture of the needle sheath with the needle tip. The needle was then used to puncture the target lesion in a standard fashion. The stylet was maximally advanced inside the needle to remove any possible tissue plugs. Following that, the stylet was either removed entirely from the needle and $10 \mathrm{~mL}$ of suction was applied, or the stylet was retracted in a "slow pull" manner, all while performing multiple to-and-fro motions of the needle within the target lesion. Use of standard stylet technique or the slow-pull technique was left to the discretion of the endoscopist.

\section{Specimen preparation and evaluation}

Fine-needle aspiration (FNA) was defined as the performance of any single needle pass in which the acquired tissue was prepared for cytologic analysis. Methods used for cytologic analysis included Diff-Quik analysis and/or the creation of a cell block analyzed by hematoxylin and eosin staining, and relevant immunohistochemical staining when appropriate. At the discretion of the endoscopist, rapid on-site evaluation (ROSE) of specimens was utilized to determine whether adequate specimen was obtained.

\begin{tabular}{|l|l|}
\hline Table 1 Patient demographics and lesion characteristics. \\
\hline Patients & $\mathrm{n}=226$ \\
\hline Median age, years & $66(18-92)$ \\
\hline Gender & Male $113(50 \%)$ \\
\hline Lesion location & $\mathrm{n}=250$ \\
\hline Subepithelial tumor & 28 \\
Esophagus & 2 \\
Stomach & 22 \\
Rectum & 3 \\
Ascending colon & 1 \\
\hline Pancreas & 136 \\
Head & 64 \\
Uncinate & 14 \\
Genu & 8 \\
Body & 25 \\
Tail & 25 \\
\hline Bile duct & 5 \\
\hline Liver & 19 \\
\hline Lymph node & 51 \\
\hline Other & 11 \\
\hline Median size $(\mathrm{mm})$ & $26(2-150)$ \\
\hline
\end{tabular}

Fine-needle biopsy (FNB) was defined as the performance of any single needle pass in which the acquired tissue was prepared for pathologic analysis. The goal of an FNB was to obtain intact tissue (i.e. a core biopsy) for histopathologic analysis. These core biopsy specimens were placed directly into formalin, embedded into paraffin, and sectioned for standard hematoxylin and eosin staning as per standard pathology protocol. Relevant immunohistochemical staining was performed as appropriate (e.g. c-kit and/ or dog-1 stain for suspected gastrointestinal stromal tumor).

Final analysis of specimens sent for cytology and/or pathology was given one of the following designations: non-diagnostic, benign, atypical, suspicious, or malignant. Both cytologic yield and pathologic yield were determined by calculating the percentage of cases receiving either a benign or malignant diagnosis; designations of atypical or suspicious were considered to be non-diagnostic in this context.

\section{Statistical analysis}

A two-sided Fisher's exact test was used to compare categorical variables. A P value $<0.05$ was considered statistically significant.

\section{Results}

\section{$\nabla$}

\section{Patient and lesion characteristics}

A total of 250 lesions underwent EUS-TA in 226 patients. Patient demographics and lesion characteristics are detailed in $\bullet$ Table 1.

\section{Technical aspects}

A 25 -gauge needle was used in $29 \%$ cases, a 22 -gauge needle was used in $65 \%$ cases, and both were used in $6 \%$ cases.

\section{Overall diagnostic yield}

A total of 194 cases had specimen sent for cytologic analysis; the median number of passes used was 3 [1 -7]. The overall cytologic diagnostic yield was 157/194 (81\%). Final cytologic diagnoses were as follows: Non-diagnostic 15 (8\%), benign 42 (22\%), atypical 10 (5\%), suspicious 12 (6\%), malignant 115 (59\%). ROSE was utilized in 149 cases. Adequate specimen as deemed by ROSE 
Table 2 Diagnostic yield by lesion type.

\begin{tabular}{|l|lllllll|}
\hline Lesion type & $\mathbf{n}$ & $\begin{array}{l}\text { Median size, } \\
\mathbf{m m} \text { (range) }\end{array}$ & $\begin{array}{l}\text { Median } \\
\text { number passes } \\
\text { for cytology } \\
\text { (range) }\end{array}$ & $\begin{array}{l}\text { Diagnostic yield } \\
\text { cytology (range) }\end{array}$ & $\begin{array}{l}\text { Median number } \\
\text { of passes for } \\
\text { pathology (range) }\end{array}$ & $\begin{array}{l}\text { Diagnostic yield, } \\
\text { pathology (n, \%) }\end{array}$ \\
\hline Pancreas & 136 & $\begin{array}{l}28 \\
(4-150)\end{array}$ & $3(1-7)$ & $85 / 106(80)$ & $2(1-10)$ & $70 / 81(86)$ & $5(2$ pancreatitis, \\
\hline Subepithelial mass & 28 & $\begin{array}{l}29 \\
(9-54)\end{array}$ & $3(1-7)$ & $19 / 28(68)$ & $2(1-5)$ & $13 / 15(87)$ \\
\hline Lymph node & 51 & $\begin{array}{l}20 \\
(2-53)\end{array}$ & $3(1-7)$ & $21 / 42(50)$ & $2(1-4)$ & $26 / 28(93)^{*}$ & $2($ pain) \\
\hline
\end{tabular}

* $P<0.05$, lymph node pathology yield vs lymph node cytology yield

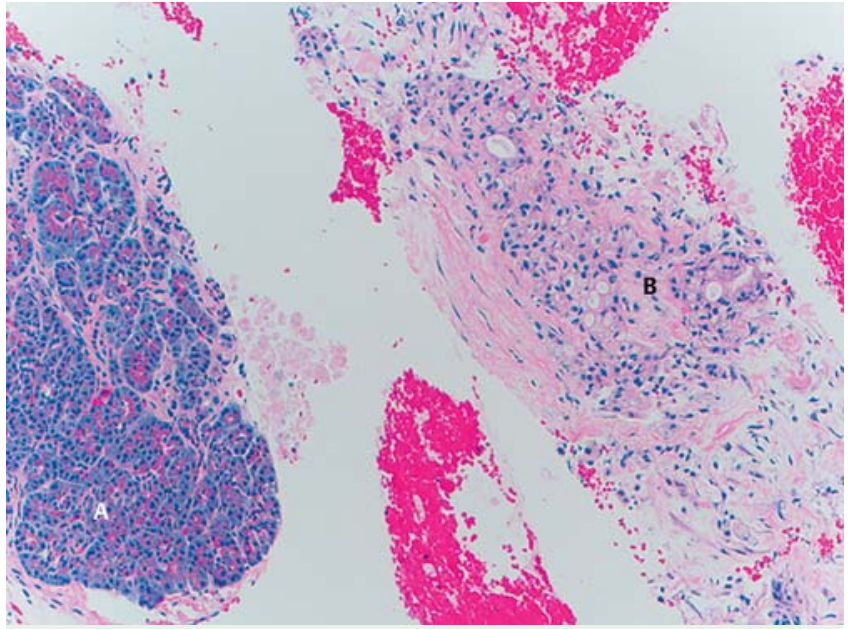

Fig. 2 Core biopsy revealing normal pancreatic tissue (a) and pancreatic adenocarcinoma (b) (medium power).

was $85 \%$. When ROSE was not utilized, the cytologic diagnostic yield was 31/45 (69\%). When ROSE was used, the cytologic diagnostic yield was $126 / 149(85 \%, P=0.03)$
A total of 147 cases had specimen sent for pathologic analysis; the median number of passes used was 2 [1-10]. The overall pathologic diagnostic yield was 130/147 (88\%, NS compared to overall cytologic yield). Final pathologic diagnoses were as follows: Non-diagnostic $7(5 \%)$, benign $30(20 \%)$, atypical $4(3 \%)$, suspicious 6 (4\%), malignant 100 (68\%).

\section{Diagnostic yield by type of lesion}

The cytologic and pathologic yields were separately calculated for pancreatic lesions, subepithelial lesions, and lymph nodes ( $\bullet$ Table 2 ) ( $\odot$ Fig. 2 and $\odot$ Fig.3). Overall pathologic yield for each lesion subtype were as follows: pancreatic lesions $86 \%$, subepithelial lesions $87 \%$, lymph nodes $93 \%$. Pathologic yield was significantly higher than cytologic yield in lymph nodes (93\% vs $50 \%$, $P=0.0002$ ).

\section{Diagnostic yield by needle size}

\section{5-gauge}

Seventy-three lesions underwent EUS-TA with a 25-gauge needle. These lesions included: subepithelial tumor (SET) 4, pancreatic mass 45 , lymph node 12 , bile duct mass 2 , liver 8 , other 2 . The median size of these lesions was $24(4-72) \mathrm{mm}$.

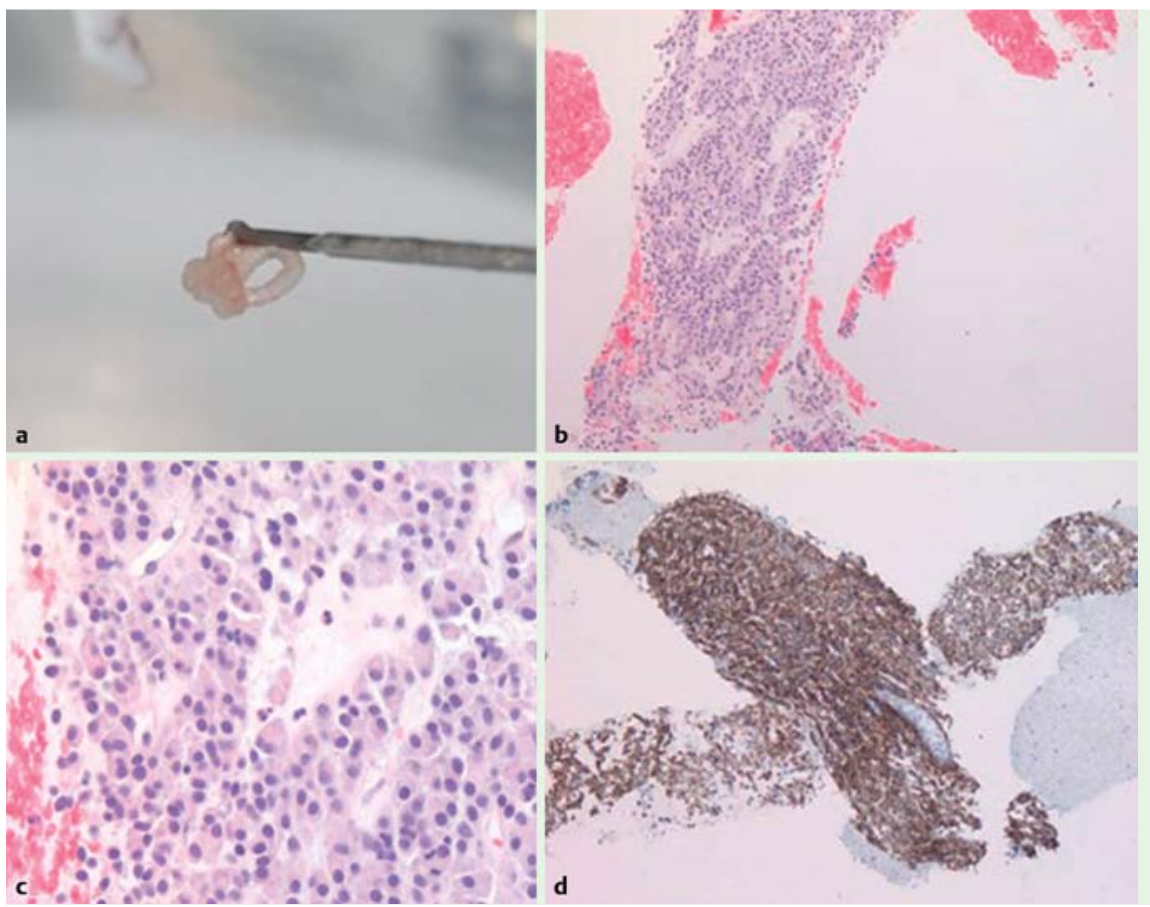

Fig. 3 Core biopsy of a gastrointestinal stromal tumor (a, b, and c), with positive c-kit staining (d). 
A total of 63 cases had specimen sent for cytology; the median number of passes sent for cytologic analysis was $3[1-7]$.The overall cytologic diagnostic yield in cases performed with a 25gauge needle was $49 / 63$ (78\%). ROSE was utilized in 49 cases. In cases in which ROSE was not utilized, the cytologic diagnostic yield was 11/14 (79\%). When ROSE was used, the cytologic diagnostic yield was 38/49 (78\%, NS).

A total of 29 cases had specimen sent for pathology; the median number of passes sent for pathologic analysis was $2[1-4]$. The overall pathologic diagnostic yield in cases performed with a 25gauge needle was $25 / 29$ ( $86 \%$, NS compared to overall cytologic yield).

\section{2-gauge}

One hundred sixty-one lesions underwent EUS-TA with a 22gauge needle. These lesions included: SET 21, pancreas 83, bile duct mass 3 , liver 11 , lymph nodes 34 , other 9 . The median size of these lesions was $27(2-150) \mathrm{mm}$.

A total of 116 cases had specimen sent for cytology; the median number of passes sent for cytologic analysis was $3(1-7)$. The overall cytologic diagnostic yield in cases performed with a 22gauge needle was 94/116 (81\%). ROSE was utilized in 88 cases. In cases in which ROSE was not utilized, the cytologic diagnostic yield was 19/28 (68\%). When ROSE was used, the cytologic diagnostic yield was $75 / 88(85 \%, P=0.05)$.

A total of 111 cases had specimen sent for pathology; 2 of these cases were liver biopsies. The overall pathologic diagnostic yield in cases performed with a 22-gauge needle was 99/111 (89\%, NS compared to overall cytologic yield).

Sixteen cases had FNA performed with both a 25 -gauge needle and a 22-gauge needle and were excluded from the above needle size analysis.

\section{Diagnostic yield by lesion type and needle size}

The cytologic and pathologic yields were separately calculated for pancreatic lesions, SET, and lymph nodes for both 25 -gauge and 22-gauge needles ( $\bullet$ Table 3 ).

\section{Adverse events}

Adverse events (AEs) were encountered in 10/250 (4\%) cases. These included post-procedure pain in 5 patients, mild acute pancreatitis in 4 patients, and fever/cholangitis in one patient 12 days after combined EUS/ERCP for a pancreatic head cancer (๑ Table 4).

\section{Discussion}

This large, multicenter retrospective analysis demonstrates that the use of a novel core biopsy needle results in excellent pathologic diagnostic yield among all lesion types with a minimum required number of needle passes.

There are a number of findings to highlight from this study. The first is the overall excellent pathologic diagnostic rate of $88 \%$ obtained with this novel needle. Previously published prospective studies examining the diagnostic yield of core biopsies obtained by using the Quick-Core (Cook Medical, Bloomington, IN, United States) biopsy needle demonstrated diagnostic yields ranging from $42 \%$ to $95 \%$, with an mean yield of $75 \%$ [3-12]. Similarly, numerous prospective studies examining the diagnostic yield of core biopsies obtained by using the Procore (Cook Medical, Bloomington, IN, United States) biopsy needle demonstrated diagnos-

\begin{tabular}{|c|c|c|}
\hline & $\begin{array}{l}\text { 25-gauge } \\
\text { n (\%) }\end{array}$ & $\begin{array}{l}\text { 22-gauge } \\
\text { n (\%) }\end{array}$ \\
\hline \multicolumn{3}{|l|}{ Pancreas } \\
\hline Cytology & $27 / 37(73)$ & $51 / 61(84)$ \\
\hline Pathology & $17 / 20(85)$ & $50 / 58(86)$ \\
\hline \multicolumn{3}{|c|}{ Subepithelial tumor } \\
\hline Cytology & $3 / 4(75)$ & $12 / 15(80)$ \\
\hline Pathology & $0 / 1(0)$ & $12 / 13(92)$ \\
\hline \multicolumn{3}{|l|}{ Lymph node } \\
\hline Cytology & $9 / 12(75)$ & $18 / 25(72)$ \\
\hline Pathology & $2 / 2(100)$ & $22 / 23(96)$ \\
\hline
\end{tabular}

\section{Table 4 Adverse events.}

\begin{tabular}{|c|c|c|c|}
\hline $\begin{array}{l}\text { Patient } \\
\text { Number }\end{array}$ & Lesion & Adverse Event & $\begin{array}{l}\text { Needle } \\
\text { gauge }\end{array}$ \\
\hline 1 & $\begin{array}{l}\text { Porta hepatis lymph } \\
\text { node }\end{array}$ & Pain & $\begin{array}{l}25-g \\
\text { and } \\
22-g\end{array}$ \\
\hline 2 & $\begin{array}{l}\text { Pancreatic head } \\
\text { cancer }\end{array}$ & Pain & $25-g$ \\
\hline 3 & $\begin{array}{l}\text { Porta hepatis lymph } \\
\text { node }\end{array}$ & Pain & $22-g$ \\
\hline 4 & $\begin{array}{l}\text { Pancreatic head } \\
\text { cancer }\end{array}$ & Pain & $22-g$ \\
\hline 5 & $\begin{array}{l}\text { Pancreatic head } \\
\text { cancer }\end{array}$ & Pain & $22-g$ \\
\hline 6 & $\begin{array}{l}\text { Pancreatic head } \\
\text { cancer }\end{array}$ & $\begin{array}{l}\text { Fever/cholangitis } 12 \text { days } \\
\text { after combined EUS/ERCP }\end{array}$ & $25-g$ \\
\hline 7 & $\begin{array}{l}\text { Chronic pancrea- } \\
\text { titis (pancreatic } \\
\text { head) }\end{array}$ & Mild acute pancreatitis & $22-g$ \\
\hline 8 & $\begin{array}{l}\text { Distal bile duct } \\
\text { mass }\end{array}$ & Mild acute pancreatitis & $22-g$ \\
\hline 9 & $\begin{array}{l}\text { Pancreatic uncinate } \\
\text { cancer }\end{array}$ & Mild acute pancreatitis & $22-g$ \\
\hline 10 & $\begin{array}{l}\text { Retroperitoneal } \\
\text { mass }\end{array}$ & Mild acute pancreatitis & $22-g$ \\
\hline
\end{tabular}

tic yields ranging from $70 \%$ to $90.2 \%$ with a mean yield of $81.1 \%$ $(13-21)$. Based on the results of our study, a randomized prospective trial comparing outcomes with the SharkCore needle versus these other needle types may answer the question about which needle design is superior in achieving diagnostic core biopsy specimens. It should be noted that core specimens for pathologic analysis can be obtained by the use of standard FNA needles. Multiple prospective studies have demonstrated high pathologic diagnostic yields ranging from $81 \%$ to $96 \%$ when using a standard 22 gauge needle and $59 \%$ to $100 \%$ when using a standard 19-gauge needle [22-29]. As such, it is unclear at this time how the SharkCore FNB needle would perform against standard FNA needles.

Pathologic yields for specific lesion subtypes included $86 \%$ for pancreatic lesions, $87 \%$ for SET, and $93 \%$ for lymph nodes. The high pathologic diagnostic yield for SET is particularly interesting, as these lesions often present a challenge for tissue acquisition by endosonographers. Published studies demonstrate a cytologic diagnostic yield ranging from $46 \%$ to $98 \%[12,28,30-$ 34]. Diagnostic yield when using the Tru-Cut biopsy needle produced pathologic diagnoses in $55 \%$ to $79 \%$ of cases, with mean number of required passes ranging from 1.9 to $4[8,12,31]$. One 
small prospective RCT comparing 22-gauge FNA needle to a 22gauge Procore needle demonstrated a significantly higher diagnostic yield with the FNB needle compared to FNA ( $75 \%$ vs $20 \%$, $P=0.01)$ and requiring significantly fewer needle passes to achieve this endpoint ( 2 vs $4, P=0.025$ ) [35]. In the current study, we demonstrated a strong trend towards a higher pathologic yield compared to cytologic yield ( $87 \%$ vs $68 \%$, NS) for EUS-TA of SET, with a median number of 2 needle passes required for core biopsy. Subgroup analysis demonstrated that use of the 22-gauge SharkCore needle resulted in both a relatively high pathologic and cytologic diagnostic yield for SET (12/13, 92\% vs $12 / 15,80 \%$, NS). As such, further studies involving a larger number of subjects may be warranted to assess the performance of the SharkCore FNB needle in EUS-TA of SET.

Another noteworthy finding from this study is that needle size did not have an impact on pathologic diagnostic yield, as both the 25-gauge needle and the 22-gauge needle performed at a very high level, $86 \%$ and $89 \%$, respectively. Future prospective studies should be designed to ascertain the effect of needle size on diagnostic outcomes. It should be noted that in regards to cytologic yield, the needle performed at an acceptable level ( $81 \%$ diagnostic yield), and that this was not significantly different compared to the overall pathologic yield (88\%). The results of this study also confirm that the utilization of ROSE has a significant impact on cytologic diagnostic yield compared to when ROSE is not used or available ( $85 \%$ vs $69 \%, P=0.03$ ), as has been demonstrated in numerous prior studies [36-38].

AEs related to the use of the SharkCore FNB needle were encountered in $10 / 250$ (4\%) of overall cases. The vast majority of AEs were encountered in patients undergoing EUS-TA of the pancreas, with 4/136 (2.9\%) experiencing mild pancreatitis, and 3/ 136 (2.2\%) experiencing pain. This AE rate is slightly higher than what would be anticipated, as EUS-TA of the pancreas is generally considered very safe, with a reported rate of pancreatitis of no more than $2 \%$ [39]. One large systematic review of more than 10,000 patients demonstrated that in patients undergoing pancreatic EUS-FNA, the rate of procedure-related pancreatitis was $0.44 \%$ and procedure-related pain was $0.34 \%$ [40]. It was noted by numerous participants in this study that the SharkCore FNB needle produced a tactile "pop" or "catch" when advanced through the tissue. It is theoretically possible that the novel design of the needle tip may allow for more effective capture of tissue prior to shearing it off, but that this may subsequently result in more localized trauma which can result in higher rates of AEs. Larger studies including more patients with pancreatic lesions should help clarify if there are in fact higher risks with using this needle in the pancreas.

There are a number of strengths and limitations to this study. This is the largest study to date examining the performance of an EUS-TA device, with 250 lesions sampled in 226 subjects.The study was a multicenter study, involving highly experienced expert endosonographers at both large academic referral centers and large, high-volume community-based practices, thus creating a real world environment to assess the performance of this novel needle. In addition, we examined outcomes related to both cytologic and pathologic diagnostic yields. The main limitation of the study is its retrospective design and the issues inherent in such studies. In particular, this study is largely subject to selection bias, as patients enrolled were non-consecutive, and it was left to the discretion of each endoscopist at each center whether any particular lesion underwent an FNA, FNB, or both, as well as whether ROSE was utilized or not. Another major lim- itation is that the cytologic and pathologic analysis was not standardized and the specimens were not centrally reviewed. This may have resulted in considerable heterogeneity in interpretation of the specimens, and greatly affected the results either positively or negatively. Another major limitation of this study is that the final diagnosis of enrolled lesions was not described. As a result, we are leaving open the possibility of both false-positive and false-negative results in our reported diagnostic yield, which could significantly impact the results of our reported outcomes. Thus, it is difficult to make comparisons of needle performance in this study with other studies. However, our intent was to examine the initial experience with this device at expert centers rather than to assert its superiority to other devices. It is our feeling that based on these results, further prospective, randomized, and controlled studies are warranted to assess needle performance and technique with other available devices.

In conclusion, this large, multicenter, North American studying examining the performance and initial experience with a novel core biopsy needle demonstrated an excellent overall pathologic diagnostic yield of $88 \%$ among all lesions types, with a minimum number of passes required to obtain diagnostic tissue. The needle performed particularly well in achieving diagnostic core biopsies of SET. There was no difference in pathologic yield between the 25 -gauge needle and the 22-gauge needle. Further prospective studies are warranted to determine if the use of this device can make the need for FNA and/or ROSE obsolete.

Competing interests: Dr. Adler is a consultant for Medtronic. Dr. Berzin is a consultant for Boston Scientific and Medtronic. Dr. DiMaio is a consultant for Boston Scientific and Medtronic. Dr. Haluszka is a consultant for Boston Scientific and Medtronic; research support from Boston Scientific. Dr. Ho is a consultant for Boston Scientific and has a speaking agreement with Cook Medical and Medtronic. Dr. Kothari is a consultant for Cook and Medtronic. Dr. Lam is on the advisory board for Boston Scientific and has received an honorarium from Medtronic. Dr. Maranki is a consultant for Boston Scientific. Dr. Muthusamy is a consultant for Boston Scientific and Medtronic and has received honoraria from Boston Scientific and Cook Medical and Medtronic and research support from Medtronic. Dr. Nieto is a consultant for Boston Scientific and Medtronic. Dr. Pleskow is a consultant for Boston Scientific and Medtronic. Dr. Ryou is a consultant for Medtronic. Dr. Sethi is a consultant for Boston Scientific and Xlumena. Dr. Sharma is a consultant for Boston Scientific and Medtronic and has received research support from Medtronic. Dr. Watson is a consultant for Medtronic. Dr. Wong is a consultant for Boston Scientific and Medtronic.

\section{Institutions}

${ }^{1}$ Dr. Henry D. Janowitz Division of Gastroenterology, Icahn School of Medicine at Mount Sinai, Mount Sinai Medical Center, New York, New York, United States

2 Mount Sinai Beth Israel Medical Center, New York, New York, United States ${ }^{3}$ Lehigh Valley Hospital, Allentown, Pennsylvania, United States

${ }^{4}$ Temple University School of Medicine, Philadelphia, Pennsylvania, United States

${ }^{5}$ University of British Columbia, Vancouver, British Columbia, Canada

${ }^{6}$ Dartmouth-Hitchcock Medical Center, Lebanon, New Hampshire, United States

7 Lahey Clinic, Burlington, Massachusetts, United States

${ }^{8}$ NewYork-Presbyterian Hospital/Columbia University, New York, New York, United States

${ }^{9}$ Borland-Groover Clinic, Jacksonville, Florida, United States

${ }^{10}$ University of Rochester Medical Center, Rochester, New York, United States

${ }^{11}$ Montefiore Medical Center, Bronx, New York, United States

12 Parkview Health System, Wayne, Indiana, United States 
${ }^{13}$ David Geffen School of Medicine at UCLA, Los Angeles, California, United States

${ }^{14}$ Beth Israel Deaconess Medical Center, Boston, Massachusetts, United States

${ }^{15}$ Brigham and Women's Hospital, Boston, Massachusetts, United States

${ }^{16}$ University of Alberta, Edmonton, Alberta, Canada

${ }^{17}$ Crystal Run Healthcare, Middletown, New York, United States

${ }^{18}$ University of Utah School of Medicine, Salt Lake City, Utah, United States

\section{References}

1 Itoi $T$, Takei $K$, Sofuni $A$ et al. Immunohistochemical analysis of $\mathrm{p} 53$ and MIB-1 in tissue specimens obtained from endoscopic ultrasonography-guided fine needle aspiration biopsy for the diagnosis of solid pancreatic masses. Oncol Rep 2005; 13: 229-234

2 Brais RJ, Davies SE, O'Donovan M et al. Direct histological processing of EUS biopsies enables rapid molecular biomarker analysis for interventional pancreatic cancer trials. Pancreatology 2012; 12: 8-15

3 Larghi A, Verna EC, Stavropoulos SN et al. EUS-guided trucut needle biopsies in patients with solid pancreatic masses: a prospective study. Gastrointest Endosc 2004; 59: 185-190

4 Wittmann J, Kocjan G, Sgouros SN et al. Endoscopic ultrasound-guided tissue sampling by combined fine needle aspiration and trucut needle biopsy: a prospective study. Cytopathology 2006; 17: 27-33

5 Aithal GP, Anagnostopoulos GK, Tam $W$ et al. EUS-guided tissue sampling: comparison of "dual sampling" (Trucut biopsy plus FNA) with "sequential sampling" (Truct biopsy and then FNA as required). Endoscopy 2007; 39: 725-730

6 Saftoiu A, Vilmann P, Guldhammer Skov B et al. Endoscopic ultrasound (EUS)-guided Trucut biopsy adds significant information to EUS-guided fine-needle aspiration in selected patients: a prospective study. Scand J Gastroenterol 2007; 42: 117-125

7 Sakamoto H, Kitano M, Komaki $T$ et al. Prospective comparative study of the EUS guided 25-gauge FNA needle with the 19-gauge Trucut needle and 22-gauge FNA needle in patients with solid pancreatic masses. J Gastroenterol Hepatol 2009; 24: $384-390$

8 Polkowski M, Gerke W, Jarosz D et al. Diagnostic yield and safety of endoscopic ultrasound-guided trucut [corrected] biopsy in patients with gastric submucosal tumors: a prospective study. Endoscopy 2009; 41: 329-334

9 Thomas T, Kaye PV, Ragunath Ket al. Efficacy, safety, and predictive factors for a positive yield of EUS-guided Trucut biopsy: a large tertiary referral center experience. Am J Gastroenterol 2009; 104: 584-591

10 Wahnschaffe $U$, Ullrich $R$, Mayerle $J$ et al. EUS-guided Trucut needle biopsies as first-line diagnostic method for patients with intestinal or extraintestinal mass lesions. Surg Endosc 2009; 23: 2351 - 2355

11 Gerke H, Rizk MK, Vanderheyden $A D$ et al. Randomized study comparing endoscopic ultrasound-guided Trucut biopsy and fine needle aspiration with high suction. Cytopathology 2010; 21: 44-51

12 DeWitt J, Emerson RE, Sherman $S$ et al. Endoscopic ultrasound-guided Trucut biopsy of gastrointestinal mesenchymal tumor. Surg Endosc 2011; 25: 2192-2202

13 Bang JY, Hebert-Magee S, Trevino J et al. Randomized trial comparing the 22-gauge aspiration and 22-gauge biopsy needles for EUS-guided sampling of solid pancreatic mass lesions. Gastrointest Endosc 2012; 76: $321-327$

14 Larghi A, Iglesias-Garcia J, Poley JW et al. Feasibility and yield of novel 22-gauge histology EUS needle in patients with pancreatic masses: a multicenter prospective cohort study. Surg Endosc 2013; 27: 37333738

15 Hucl T, Wee E, Anuradha $S$ et al. Feasibility and efficiency of a new $22 \mathrm{G}$ core needle: a prospective comparison study. Endoscopy 2013; 45: $792-798$

$16 \mathrm{Kim}$ GH, Cho YK, Kim EY et al. Comparison of 22-gauge aspiration needle with 22-gauge biopsy needle in endoscopic ultrasonography-guided subepithelial tumor sampling. Scand J Gastroenterol 2014; 49: $347-354$

17 Lee YN, Moon JH, Kim HK et al. Core biopsy needle versus standard aspiration needle for endoscopic ultrasound-guided sampling of solid pancreatic masses: a randomized parallet group study. Endoscopy 2014; 46: 1056-1062

18 Mavrogenis G, Weynand B, Sibille A et al. 25-gauge histology needle versus 22 -gauge cytology needle in endoscopic ultrasonography-guided sampling of pancreatic lesions and iglelymphadenopathy. Endosc Int Open 2015; 3: E63-E68
19 Strand DS, Jeffus SK, Sauer BG et al. EUS-guided 22-gauge fine-needle aspiration versus core biopsy needle in the evaluation of solid pancreatic neoplasms. Diagn Cytopathol 2014; 42: 751 -758

20 Vanbiervliet G, Napoleon B, Paul MCS et al. Core needle versus standard needle for endoscopic ultrasound-guided biopsy of solid pancreatic masses: a randomized crossover study. Endoscopy 2014; 46: $1063-$ 1070

21 Bang JY, Hawes R, Varadarajulu S. A meta-analysis comparing ProCore and standard fine-needle aspiration needles for endoscopic ultrasound-guided tissue acquisition. Endoscopy 2016; 48: 339-349

22 Voss M, Hammel P, Molas G et al. Value of endoscopic ultrasound guided fine needle aspiration biopsy in the diagnosis of solid pancreatic masses. Gut 2000; 46: 244-249

23 Larghi A, Noffsinger A, Dye CE et al. EUS-guided fine needle tissue acquisition by using high negative pressure suction for the evaluation of solid masses: a pilot study. Gastrointest Endosc 2005; 62: 768 - 774

24 Yasuda I, Tsurumi H, Omar $S$ et al. Endoscopic ultrasound-guided fine needle aspiration biopsy for lymphadenopathy of unknown origin. Endoscopy 2006; 38: 919-924

25 Iglesias-Garcia J, Dominguez-Munoz E, Lozano-Leon A et al. Impact of endoscopic ultrasound-guided fine needle aspiration biopsy for diagnosis of pancreatic masses. World J Gastroenterol 2007; 13: 289-293

26 Larghi A, Verna EC, Ricci R et al. EUS-guided fine-needle tissue acquisition by using a 19-gauge needle in a selected patient population: a prospective study. Gastrointest Endosc 2011; 74: 504-510

27 Varadarajulu S, Bang JY, Hebert-Magee S. Assessment of the technical performance of the flexible 19-gauge EUS-FNA needle. Gastrointest Endosc 2012; 76: $336-343$

28 Eckardt AJ, Adler A, Gomes EM et al. Endosonographic large-bore biopsy of gastric subepithelial tumors: a prospective multicenter study. Eur J Gastroenterol Hepatol 2012; 24: 1135-1144

29 Al-Haddad MA, Aggarwal A, Arnan A. EUS-Guided Core Biopsy with a novel 19-Gauge Flexible Fine Needle Biopsy (FNB) Device: multicenter experience. Gastrointest Endosc 2013; 77: AB403-AB404

30 Philipper M, Hollerbach S, Gabbert HE et al. Prospective comparison of endoscopic ultrasound-guided fine-needle aspiration and surgical histology in upper gastrointestinal submucosal tumors. Endoscopy 2010; 42: $300-305$

31 Fernandez-Esparrach $G$, Sendino $O$, Sole $M$ et al. Endoscopic ultrasoundguided fine-needle aspiration and trucut biopsy in the diagnosis of gastric stromal tumors: a randomized crossover study. Endoscopy 2010; 42: 292-299

32 Yoshida S, Yamashita K, Yokozawa $M$ et al. Diagnostic findings of ultrasound-guided fine-needle aspiration cytology for gastrointestinal stromal tumors: proposal of a combined cytology with newly defined features and histology diagnosis. Pathol Int 2009; 59: 712-719

33 Akahoshi K, Sumida Y, Matsui $N$ et al. Preoperative diagnosis of gastrointestinal stromal tumor by endoscopic ultrasound-guided fine needle aspiration. World J Gastroenterol 2007; 13: 2077-2082

34 Ando N, Goto H, Niwa Y et al. The diagnosis of GI stromal tumors with EUS-guided fine needle aspiration with immunohistochemical analysis. Gastrointest Endosc 2002; 55: 37-43

35 Chen VK, Eloubeidi MA. Endoscopic ultrasound-guided fine-needle aspiration of intramural and extraintestinal mass lesions: diagnostic accuracy, complication assessment, and impact on management. Endoscopy 2005; 37: 984-989

36 Iglesias-Garcia J, Dominguez-Munoz JE, Abdulkader I et al. Influence of on-site cytopathology evaluation on the diagnostic accuracy of endoscopic ultrasound-guided fine needle aspiration. Am J Gastroenterol 2011; 106: 091705-1710

37 Klapman JB, Logrono R, Dye CE et al. Clinical impact of on-site cytopathology interpretation on endoscopic ultrasound-guided fine needle aspiration. Am J Gastroenterol 2003; 98: 1289-1294

38 Ecka RS, Sharma M. Rapid on-site evaluation of EUS-FNA by cytopathologist: an experience of a tertiary hospital. Diagn Cytopathol 2013; 41: $1075-1080$

39 Early DS, Acosta RD, Chandrasekhara V et al. Adverse events associated with EUS and EUS with FNA. Gastrointest Endosc 2013; 77: 839-843

40 Wang KX, Ben QW, Jin ZD et al. Assessment of morbidity and mortality associated with EUS-guided FNA: a systematic review. Gastrointest Endosc 2011; 73: 283-290 